\title{
A ZERO DIVISOR GRAPH DETERMINED BY EQUIVALENCE CLASSES OF ZERO DIVISORS
}

\author{
SANDRA SPIROFF AND CAMERON WICKHAM
}

\begin{abstract}
We study the zero divisor graph determined by equivalence classes of zero divisors of a commutative Noetherian ring $R$. We demonstrate how to recover information about $R$ from this structure. In particular, we determine how to identify associated primes from the graph.
\end{abstract}

\section{INTRODUCTION}

Beck first introduced the notion of a zero divisor graph of a ring $R$ in 1988 [4] from the point of view of colorings. Since then, others have studied and modified these graphs, whose vertices are the zero divisors of $R$, and found various properties to hold. Inspired by ideas from S. Mulay in [7, §3], we introduce the graph of equivalence classes of zero-divisors of a ring $R$, which is constructed from classes of zero divisors determined by annihilator ideals, rather than individual zero divisors themselves. It will be denoted by $\Gamma_{E}(R)$.

This graph has some advantages over the earlier zero divisor graphs $\Gamma(R)$ in $[4$, [1], 2], 3], or subsequent zero divisor graphs determined by an ideal of $R$ in [5], 8]. In many cases $\Gamma_{E}(R)$ is finite when $\Gamma(R)$ is infinite. For example, if $S=\mathbb{Z}[X, Y] /\left(X^{3}, X Y\right)$, then $\Gamma(S)$ is an infinite graph, while $\Gamma_{E}(S)$ has only four vertices. To be specific, although $X^{2}, 2 X^{2}, 3 X^{2}, \ldots$, are distinct zero divisors, they all have the same annihilator; they are represented by a single vertex in $\Gamma_{E}(S)$. In addition, there are no complete $\Gamma_{E}(R)$ graphs with three or more vertices since the graph would collapse to a single point. These are two ways in which $\Gamma_{E}(R)$ represents a more succinct description of the "zero divisor activity" in $R$.

Another important aspect of graphs of equivalence classes of zero divisors is the connection to associated primes of the ring. For example, in the ring $S$ above, the annihilator of $X^{2}$ is an associated prime. In general, all of the associated primes of a ring $R$ correspond to distinct vertices in $\Gamma_{E}(R)$. Moreover, every vertex in a graph either corresponds to an associated prime or is connected to one. The study of the structure of associated primes in $\Gamma_{E}(R)$ is one of our main motivations.

In section one, we compare and contrast $\Gamma_{E}(R)$ with the more familiar $\Gamma(R)$ defined by D. Anderson and P. Livingston [1]. In section two, we consider infinite graphs and fan graphs and answer the question of whether or not the Noetherian condition on $R$ is enough to force $\Gamma_{E}(R)$ to be finite. Section three is devoted to the relation between the associated primes of $R$ and the vertices of $\Gamma_{E}(R)$. In particular, we demonstrate how to identify some elements of $\operatorname{Ass}(R)$.

1991 Mathematics Subject Classification. Primary 13A15, 13A99, 05 C12. 
Throughout, $R$ will denote a commutative Noetherian ring with unity, and all graphs are simple graphs in the sense that there are no loops or double edges.

\section{Definitions and Basic Results}

Let $Z^{*}(R)$ denote the zero divisors of $R$ and $Z(R)=Z^{*}(R) \cup\{0\}$. For $x, y \in R$, we say that $x \sim y$ if and only if $\operatorname{ann}(x)=\operatorname{ann}(y)$. As noted in [7, $\sim$ is an equivalence relation. Furthermore, if $x_{1} \sim x_{2}$ and $x_{1} y=0$, then $y \in \operatorname{ann}\left(x_{1}\right)=\operatorname{ann}\left(x_{2}\right)$ and hence $x_{2} y=0$. It follows that multiplication is well-defined on the equivalence classes of $\sim$; that is, if $[x]$ denotes the class of $x$, then the product $[x] \cdot[y]=[x y]$ makes sense. Note that $[0]=\{0\}$ and $[1]=R-Z(R)$; the other equivalence classes form a partition of $Z^{*}(R)$.

Definition 1.1. The graph of equivalence classes of zero-divisors of a ring $R$, denoted $\Gamma_{E}(R)$, is the graph associated to $R$ whose vertices are the classes of elements in $Z^{*}(R)$, and with each pair of distinct classes $[x],[y]$ joined by an edge if and only if $[x] \cdot[y]=0$.

Recall that a prime ideal $\mathfrak{p}$ of $R$ is an associated prime if $\mathfrak{p}=\operatorname{ann}(y)$ for some $y \in R$. The set of associated primes is denoted $\operatorname{Ass}(R)$; it is well known that for a Noetherian ring $R, \operatorname{Ass}(R)$ is finite, and any maximal element of the family of ideals $\mathfrak{F}=\{\operatorname{ann}(x) \mid 0 \neq x \in R\}$ is an associated prime. There is a natural injective map from $\operatorname{Ass}(R)$ to the vertex set of $\Gamma_{E}(R)$ given by $\mathfrak{p} \mapsto[y]$ where $\mathfrak{p}=\operatorname{ann}(y)$. As a result, we will slightly abuse terminology and refer to $[y]$ as an associated prime.

Lemma 1.2. Any two distinct elements of $\operatorname{Ass}(R)$ are connected by an edge. Furthermore, every vertex $[v]$ of $\Gamma_{E}(R)$ is either an associated prime or adjacent to an associated prime maximal in $\mathfrak{F}$.

Proof. The proof of the first statement is essentially the same as in [3, Lemma 2.1]. If $\mathfrak{p}=\operatorname{ann}(x)$ and $\mathfrak{q}=\operatorname{ann}(y)$ are primes, then one can assume that there is an element $r \in \mathfrak{p} \backslash \mathfrak{q}$. Since $r x=0 \in \mathfrak{q}, x \in \operatorname{ann}(y)$, and hence $[x] \cdot[y]=0$. Next, suppose $[v] \in \Gamma_{E}(R)$ is not an associated prime. Since $v$ is a zero divisor, $v \in \operatorname{ann}(z)$ for some $z$ maximal in $\mathfrak{F}$; thus, there is an edge between $[v]$ and $[\mathrm{z}]$.

Example 1.3. The associated primes of $R=\mathbb{Z}_{4} \times \mathbb{Z}_{4}$ are $[(2,0)]$ and $[(0,2)]$.

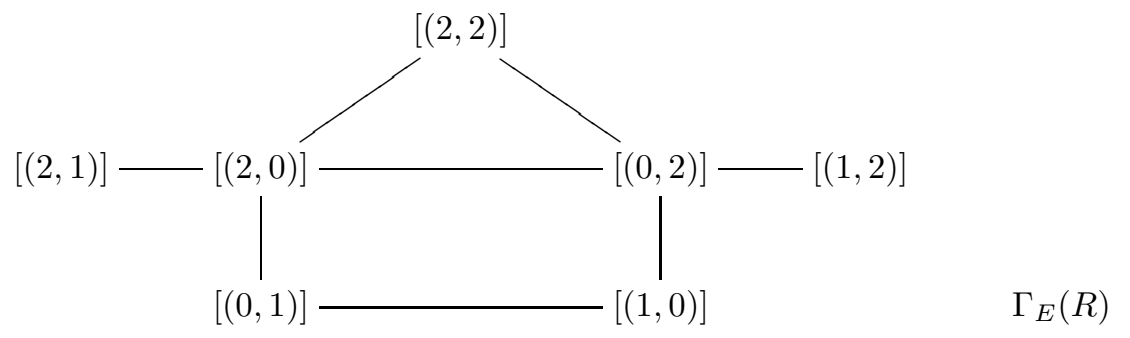

The degree of a vertex $v$ in a graph, denoted $\operatorname{deg} v$, is the number of edges incident to $v$. When $\operatorname{deg} v=1$, we call $v$ a leaf. A path of length $n$ between two vertices $u$ and $w$ is a sequence of distinct vertices $v_{i}$ of the form $u=v_{0}-v_{1}-\ldots-v_{n}=w$ such that $v_{i-1}-v_{i}$ is an edge for each $i=1, \ldots, n$. The distance between a pair of vertices is the length of the shortest path between them; if no path exists the 
distance is infinite. The diameter of a graph is the greatest distance between any two distinct vertices.

Proposition 1.4. The graph $\Gamma_{E}(R)$ is connected and diam $\Gamma_{E}(R) \leq 3$.

Proof. Let $[x]$ and $[y]$ be two non-adjacent vertices. At worst, neither $[x]$ nor $[y]$ is an associated prime. By Lemma 1.2 , there are associated primes $\left[v_{1}\right]$ and $\left[v_{2}\right]$ adjacent to $[x]$ and $[y]$, respectively, providing a path $[x]-\left[v_{1}\right]-\left[v_{2}\right]-[y]$, where $\left[v_{1}\right]$ may or may not equal $\left[v_{2}\right]$. In any case, $\Gamma_{E}(R)$ is connected and $\operatorname{diam} \Gamma_{E}(R) \leq 3$.

Connectivity and a restricted diameter are two similarities between $\Gamma_{E}(R)$ and $\Gamma(R)$, 1]. One important difference between $\Gamma_{E}(R)$ and $\Gamma(R)$ lies in the study of complete graphs. The idea in the next proposition is that complete zero divisor graphs determined by equivalence classes, with the exception of graphs consisting of a single edge (e.g., $\Gamma_{E}\left(\mathbb{Z}_{p q}\right) ; \Gamma_{E}\left(\mathbb{Z}_{p^{3}}\right)$ ), all collapse to a single point. Each vertex in $\Gamma_{E}(R)$ is representative of a distinct class of zero divisor activity in $R$.

Proposition 1.5. Let $R$ be such that $\Gamma_{E}(R)$ has at least three vertices. Then $\Gamma_{E}(R)$ is not complete.

Proof. Suppose $\Gamma_{E}(R)$ is a complete graph and $[x],[y]$, and $[z]$ are three distinct vertices. We may assume that $\operatorname{ann}(z)$ is a maximal element of $\mathfrak{F}$ and hence is not contained in either $\operatorname{ann}(x)$ or $\operatorname{ann}(y)$. Then there is an element $w \in \operatorname{ann}(z)$ such that $w \notin \operatorname{ann}(x) \cup \operatorname{ann}(y)$. This immediately contradicts the assumption that the graph is complete.

Corollary 1.6. There is only one graph $\Gamma_{E}(R)$ with exactly three vertices that can be realized as a graph of equivalence classes of zero divisors for some ring $R$.

Proof. Since every graph is connected, by Proposition 1.5, we need only note that the graph of $R=\mathbb{Z}_{4}[X, Y] /\left(X^{2}, X Y, 2 X\right)$, for example, is [2] - $[x]-[y]$, where $x$ and $y$ denote the images of $X$ and $Y$, respectively.

The simplification, or collapse, of complete $\Gamma_{E}(R)$ graphs applies also to complete $r$-partite diagrams, with the exclusion of fan graphs $K_{n, 1}$, like the one above. (The latter are considered in the next section.) A graph is complete bipartite if there is a partition of the vertices into two subsets $\left\{u_{i}\right\}$ and $\left\{v_{j}\right\}$ such that $u_{i} v_{j}=0$ for all pairs $i, j$, but no two elements of the same subset annihilate one another. More generally, a graph is complete $r$-partite if the vertices can be partitioned into $r$ distinct subsets such that each element of a subset is connected to every element not in the same subset, but no two elements of the same subset are connected.

Proposition 1.7. Let $R$ be a ring such that $\Gamma_{E}(R)$ is complete $r$-partite. Then $r=2$ and $\Gamma_{E}(R)=K_{n, 1}$ for some $n \geq 1$.

Proof. First suppose $\Gamma_{E}(R)=K_{n_{1}, n_{2}, \ldots, n_{r}}$ for some $r \geq 3$. Proposition 1.5 implies that not all $n_{i}=1$; without loss of generality assume $n_{1}>1$ and let $\left[u_{1}\right],\left[u_{2}\right]$ be two non-adjacent vertices. Since $\left[u_{1}\right] \neq\left[u_{2}\right]$, there exists $z \in \operatorname{ann}\left(u_{1}\right) \backslash \operatorname{ann}\left(u_{2}\right)$. But $\left[u_{1}\right]$ and $\left[u_{2}\right]$ have the same set of neighbors, hence $\operatorname{ann}\left(u_{1}\right) \backslash\left\{u_{1}\right\}=\operatorname{ann}\left(u_{2}\right) \backslash\left\{u_{2}\right\}$, forcing $z=u_{1}$ and hence $u_{1}^{2}=0$. Let $[v]$ and $[w]$ be two adjacent vertices of $\Gamma_{E}(R)$ both of which are adjacent to $\left[u_{1}\right]$ and $\left[u_{2}\right]$. If $v^{2}=0$, then $\left[u_{1}+v\right]$ is adjacent to 
$\left[u_{1}\right]$ but not $\left[u_{2}\right]$, a contradiction. A similar contradiction is reached if $w^{2}=0$. If $v^{2} \neq 0$ and $w^{2} \neq 0$, then $[v+w]$ is not adjacent to either $[v]$ or $[w]$, a contradiction. Thus $r=2$.

If $n_{1}>1$ and $n_{2}>1$, then there are two pairs of non-adjacent vertices, say [ $\left.u_{1}\right],\left[u_{2}\right]$ and $\left[v_{1}\right],\left[v_{2}\right]$. Arguing as above, we have $u_{1}^{2}=0=v_{1}^{2}$. But then $\left[u_{1}+v_{1}\right]$ is adjacent to both $\left[u_{1}\right]$ and $\left[v_{1}\right]$, a contradiction. Thus either $n_{1}=1$ or $n_{2}=1$ and hence $\Gamma_{E}(R)=K_{n, 1}$.

A second look at the above results allows us to deduce some facts about cycle graphs, which are $n$-gons. An immediate consequence of Proposition 1.4 is that there are no cycle graphs with eight or more vertices. Likewise, Proposition 1.7 tells us that there are no 3 - or 4 -cycle graphs, i.e., $K_{1,1,1}$ or $K_{2,2}$, respectively.

Proposition 1.8. For any $R, \Gamma_{E}(R)$ is not a cycle graph.

Proof. As mentioned above, it suffices to show that cycle graphs which contain a path of length five are not possible. Suppose $[x]-[y]-[z]-[w]-[v]$ is a path in the graph. Then $[y w]$ is annihilated by $[x],[z]$, and $[v]$, but no such class exists.

For a vertex $v$ of a simple graph $G$, the set of vertices adjacent to $v$ is called the neighborhood of $v$ and denoted $N_{G}(v)$, or simply $N(v)$ if the graph is understood.

Lemma 1.9. Let $G$ be a finite, simple graph with the property that two distinct vertices $v$ and $w$ of $G$ are non-adjacent if and only if $N_{G}(v)=N_{G}(w)$. Then $G$ is a complete r-partite graph for some positive integer $r$.

Proof. We induct on the number of vertices of $G$. If $G$ has one vertex, then $G$ is a complete 1-partite graph. So suppose $G$ has more than one vertex. Let $I=$ $\left\{y_{1}, y_{2}, \ldots, y_{n}\right\}$ be a maximal set of pairwise non-adjacent vertices in $G$ (that is, $I$ is an independent set of $G$ ), and let $N=\left\{x_{1}, \ldots, x_{d}\right\}$ be the common neighborhood of the elements of $I$. Then $I$ and $N$ are disjoint and the vertices of $G$ are precisely $I \cup N$. If $N=\varnothing$, then $G$ is 1-partite and we are done. So assume $N \neq \varnothing$. Let $H$ be the graph induced by $N$. Note that $N_{H}(v)=N_{G}(v) \cap N$ and $N_{G}(v)=N_{H}(v) \cup I$ for any vertex $v$ of $H$, hence two distinct vertices $v$ and $w$ of $H$ are non-adjacent if and only if $N_{H}(v)=N_{H}(w)$. Since $H$ has fewer vertices than $G$, the induction hypothesis implies that $H$ is a complete $(r-1)$-partite graph for some $r \geq 2$. But by the definition of $I$, this implies that $G$ is a complete $r$-partite graph.

Proposition 1.10. For any ring $R$, there are no finite regulan graphs $\Gamma_{E}(R)$ with more than two vertices.

Proof. Suppose that $R$ is a ring such that $\Gamma_{E}(R)$ is a regular graph of degree $d$ with at least 3 vertices. By earlier results, $d \geq 2$ and $|N([x])|=d$ for all $[x] \in \Gamma_{E}(R)$. Since $\Gamma_{E}(R)$ is not complete, there exists two non-adjacent vertices $\left[y_{1}\right]$ and $\left[y_{2}\right]$. If $N\left(\left[y_{1}\right]\right) \neq N\left(\left[y_{2}\right]\right)$, then without loss of generality we may assume that there is a vertex $[u] \in N\left(\left[y_{1}\right]\right) \backslash N\left(\left[y_{2}\right]\right)$. Thus $u y_{1}=0$ but $u y_{2} \neq 0$. This implies $[u] \in$ $N\left(\left[y_{1} y_{2}\right]\right)$ and so $N\left(\left[y_{2}\right]\right) \neq N\left(\left[y_{1} y_{2}\right]\right)$. But $N\left(\left[y_{2}\right]\right) \subseteq N\left(\left[y_{1} y_{2}\right]\right)$ and since each set has cardinality $d$ we must have equality, which leads to a contradiction. Therefore,

\footnotetext{
${ }^{1} \mathrm{~A}$ regular graph is one in which $\operatorname{deg} v=\operatorname{deg} w$ for every pair of vertices $v$ and $w$ of the graph.
} 
any two non-adjacent vertices on the graph have the same neighborhood, and clearly the converse is true. Thus by Lemma 1.9 and Proposition 1.7. $\Gamma_{E}(R)=K_{n, 1}$ for some $n \geq 1$. If $\mathcal{I}=\left\{\left[y_{1}\right],\left[y_{2}\right], \ldots,\left[y_{n}\right]\right\}$ is a maximal independent set in $\Gamma_{E}(R)$ and $\mathcal{N}=\left\{\left[x_{1}\right], \ldots,\left[x_{d}\right]\right\}$ is the common neighborhood of the elements of $\mathcal{I}$, then $n \geq 2$ and by (the proof of) Lemma 1.9, $\mathcal{I}$ is one of the partitions of the vertex set of $\Gamma_{E}(R)$. This implies that $d=1$, leading to a contradiction. Thus $\Gamma_{E}(R)$ is not regular.

Finally, we give the connection between $\Gamma_{E}(R)$ and the zero-divisor graph $\Gamma(R)$ in 1]. Let $\Gamma$ be a graph. To each vertex $v_{i}$ of $\Gamma$, assign an element $w_{i} \in \mathbb{Z}^{+} \cup\{\infty\}$, called the weight of $v_{i}$, and let $w=\left(w_{1}, w_{2}, \ldots\right)$. Define a graph $\Gamma^{(w)}$ with vertex set $\left\{v_{k_{i}, i} \mid 1 \leq k_{i} \leq w_{i}\right\}$ and edge set is $\left\{v_{k_{i}, i} v_{k_{j}, j} \mid i \neq j\right.$ and $v_{i} v_{j}$ is an edge of $\left.\Gamma\right\}$. Intuitively, the vertices $v_{k_{i}, i}$ of $\Gamma^{(w)}$ form a "covering set" of cardinality $w_{i}$ of the vertex $v_{i}$ of $\Gamma$, and if $v_{i}$ is connected to $v_{j}$ in $\Gamma$, then every vertex of the covering set of $v_{i}$ is connected to every vertex of the covering set of $v_{j}$.

For each vertex $\left[v_{i}\right]$ of $\Gamma_{E}(R)$, let $w_{i}=\left|\left[v_{i}\right]\right|$, and let $w=\left(w_{1}, w_{2}, \ldots\right)$. By the definitions of $\Gamma_{E}(R)$ and $\Gamma(R)$, it is clear that $\Gamma_{E}(R)^{(w)}$ is a subgraph of $\Gamma(R)$. In fact, $\Gamma_{E}(R)^{(w)}=\Gamma(R)$ if and only if whenever $R$ contains a non-zero element $x$ such that $x^{2}=0$, then $|[x]|=1$. In particular, if $R$ is reduced, then $\Gamma_{E}(R)^{(w)}=\Gamma(R)$. In general, $\Gamma(R)$ is the graph obtained from $\Gamma_{E}(R)^{(w)}$ by adding edges between every pair of vertices $v_{k_{i}, i}$ and $v_{k_{i}^{\prime}, i}$ if and only if $v_{i}^{2}=0$.

Example 1.11. The zero divisor graph $\Gamma\left(\mathbb{Z}_{12}\right)$ is shown "covering" the graph $\Gamma_{E}\left(\mathbb{Z}_{12}\right)$. Note that if $w=(2,1,2,2)$, then $\Gamma_{E}\left(\mathbb{Z}_{12}\right)^{(w)}=\Gamma\left(\mathbb{Z}_{12}\right)$.

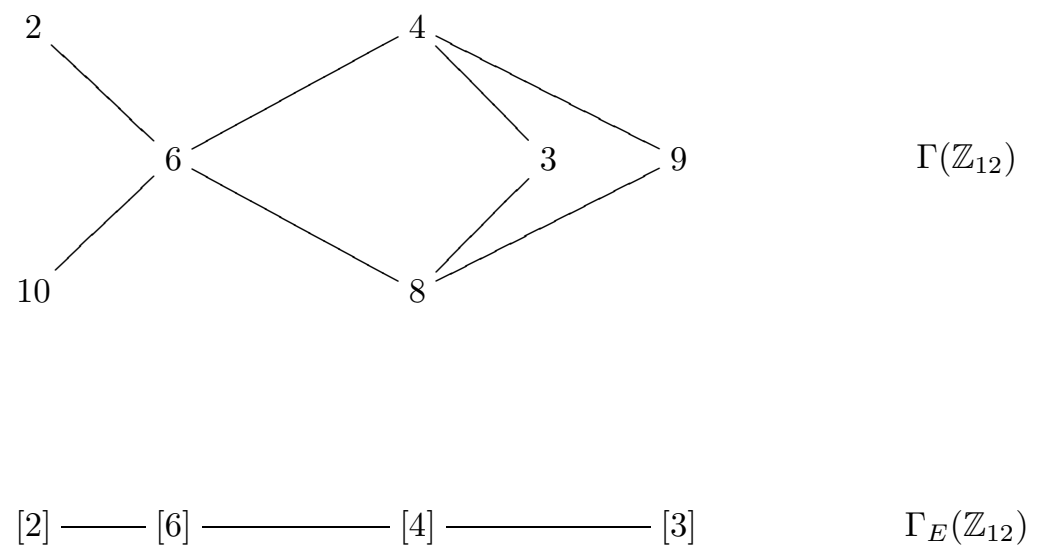

\section{Infinite Graphs and FAn Graphs}

In this section we investigate the properties of infinite graphs. There are ostensibly two ways a graph can be infinite, namely it can have infinitely many vertices or it can have vertices with infinite degree. More importantly, one might ask if the assumption that $R$ is Noetherian forces $\Gamma_{E}(R)$ to be a finite graph. We begin with a discussion of degrees.

Proposition 2.1. Let $x$ and $y$ be elements of $R$. If $0 \neq \operatorname{ann}(x) \subsetneq \operatorname{ann}(y)$, then $\operatorname{deg}[x] \leq \operatorname{deg}[y]$ 
Proof. If $[u] \in \Gamma_{E}(R)$ such that $u x=0$, then clearly $u y=0$.

Proposition 2.2. The vertex set of $\Gamma_{E}(R)$ is infinite if and only if there is some associated prime $[x]$ maximal in $\mathfrak{F}$ such that $\operatorname{deg}[x]=\infty$.

Proof. Clearly, if some vertex has infinite degree, then the vertex set of $\Gamma_{E}(R)$ is infinite. Conversely, suppose the vertex set of $\Gamma_{E}(R)$ is infinite. Let $\left[x_{1}\right], \ldots,\left[x_{r}\right]$ be the maximal elements in $\mathfrak{F}$. If $\operatorname{deg}\left[x_{1}\right]<\infty$, then there are infinitely many vertices $[w]$ such that $w x_{1} \neq 0$. Now $[w][v]=0$ for some class $[v] \neq[w]$, and since $\left[x_{1}\right]$ is prime, we must have $v \in \operatorname{ann}\left(x_{1}\right)$. If there are infinitely many distinct vertices $[v]$, then $\operatorname{deg}\left[x_{1}\right]=\infty$, a contradiction. Therefore, the set of $[v]$ 's is finite, and hence $\operatorname{deg}[v]=\infty$ for some $v$. Either $[v]$ is an associated prime of $R$ and maximal in $\mathfrak{F}$, or $\operatorname{ann}(v) \subsetneq \operatorname{ann}\left(x_{j}\right)$ for some $j \neq 1$. In either case, the result holds.

Definition 2.3. A fan graph is a complete bipartite graph $K_{n, 1}, n \in \mathbb{N} \cup\{\infty\}$. If $n=\infty$, we say the graph is an infinite fan graph.

Proposition 2.4. Any ring $R$ such that $\Gamma_{E}(R)$ is a fan graph with at least four vertices satisfies the following properties:

(1) $\operatorname{Ass}(R)=\{\mathfrak{p}\}$

(2) $\mathfrak{p}^{3}=0$

(3) $\operatorname{char}(R)=2,4$, or 8 .

Proof. Suppose we have a fan graph with at least four vertices, as show below.

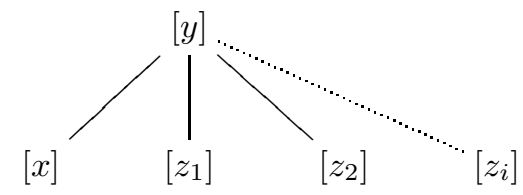

Let $[y]$ be the unique vertex with maximal degree. Note that $[y]$ is a maximal element in $\mathfrak{F}$, for if $\operatorname{ann}(y) \subsetneq \operatorname{ann}(w)$ for some $w$, then by Proposition $2.1 \Gamma_{E}(R)$ would contain two vertices of degree larger than 1 . Next, in order for the classes $[x],\left[z_{1}\right], \ldots$ to be distinct, we must have $z_{i}^{2}=0$, for all $i$. If $i \neq j$, then $z_{i} z_{j}$ is nonzero and annihilated by $z_{i}$ and $z_{j}$; hence, the only choice for $\left[z_{i} z_{j}\right]$ is $[y]$. Moreover, $\left(z_{i} z_{j}\right)^{2}=0$ implies that every element of $[y]$ is nilpotent of order 2, by [7, (3.5)]. Since $[y]$ is connected to every vertex, it follows that $z_{i} z_{j} z_{k}=z_{i} z_{j} x=0$ for any $i, j, k$. As a result, we have $\left[x z_{i}\right]=[y]$. Thus, $x^{2} z_{i}=0$ for each $i$. Consequently, either $x^{2}=0$ or $\left[x^{2}\right]=[y]$. Since the latter case implies $x^{3}=0$, it follows that every zero divisor is nilpotent of order at most three. This establishes (1) and (2).

For (3), consider $z_{i}+z_{j}$, which is annihilated by $z_{i} z_{j}$, but not $z_{i}$ or $z_{j}$. Therefore, $\left[z_{i}+z_{j}\right]$ represents a degree one vertex distinct from $\left[z_{i}\right]$ and $\left[z_{j}\right]$. The same conclusion can be reached for $\left[z_{i}-z_{j}\right]$. Since $\left(z_{i}+z_{j}\right)\left(z_{i}-z_{j}\right)=0$, this means that $\left[z_{i}-z_{j}\right]=\left[z_{i}+z_{j}\right]$, and every element in the class is nilpotent of order two. In particular, $\left(z_{i}+z_{j}\right)^{2}=0 \Rightarrow 2 z_{i} z_{j}=0$. Consequently, either $2 \equiv 0$ or 2 is a zero divisor in $R$. If $\operatorname{char}(R) \neq 2$, then [2] is somewhere on the graph and either $2^{2}=0$ or $2^{3}=0$.

Corollary 2.5. If $R$ is a finite ring and $\Gamma_{E}(R)$ is a fan graph with at least four vertices, then $R$ is a local ring. 
Proof. A finite ring is a product of finite local rings, and the number of associated primes corresponds to the number of factors in the product; see, e.g., 6].

It is unknown to the authors whether, for each positive integer $n$, the fan graph $K_{n, 1}$ can be realized as $\Gamma_{E}(R)$ for some ring $R$, or how one would go about the general construction or argument. However, the following example shows that there exist rings $R$ such that $\Gamma_{E}(R)$ is a fan graph with infinitely many leaves.

Example 2.6. Let $R=\mathbb{Z}_{2}[X, Y, Z] /\left(X^{2}, Y^{2}\right)$. Then $\Gamma_{E}(R)$ is an infinite fan graph. If $x$ and $y$ denote the images of $X$ and $Y$, respectively, then $\operatorname{Ass}(R)=\{(x, y)\}$, and the corresponding vertex $[x y]$ is the central vertex of the graph. The leaves, besides $[x],[y]$, are vertices of the form $\left[z^{m} x+z^{n} y\right]$ for each ordered pair $(m, n)$ of nonnegative integers.

Remark 2.7. Example 2.6 shows that the Noetherian condition is not enough to force $\Gamma_{E}(R)$ to be finite.

\section{Associated Primes}

One of the main motivations in studying graphs of equivalence classes of zero divisors is the fact that the associated primes of $R$, by their very definition, correspond to vertices in $\Gamma_{E}(R)$. The focus here is the identification of associated primes of $R$, given $\Gamma_{E}(R)$.

It is important to note from Proposition 2.1 in the last section that proper containment of annihilator ideals does not translate into a strict inequality of degrees. For instance, the ring $\mathbb{Z}[X, Y] /\left(X^{3}, X Y\right)$, shown in Example 3.5 below, satisfies $\operatorname{ann}(y) \subsetneq \operatorname{ann}(x)$, but $\operatorname{deg}[y]=\operatorname{deg}[x]=2$. In order to achieve strict inequality on degrees, further assumptions on the annihilator ideals are needed. This issue is addressed in this section; but first we make some key observations. The contrapositive of Proposition 2.1 is useful and worth stating:

Proposition 3.1. Let $x$ and $y$ be elements of $R$. If $\operatorname{deg}[y]>\operatorname{deg}[x]$ for every $[x]$ in $\Gamma_{E}(R)$, then $\operatorname{ann}(y)$ is maximal in $\mathfrak{F}$ and hence is an associated prime.

Of course, we already saw evidence of this fact in Proposition 2.4. In that case, the vertex of maximal degree corresponds to the unique element of $\operatorname{Ass}(R)$. Similarly, if $\Gamma_{E}(R)$ has exactly three vertices, then the vertex of degree two always corresponds to an associated prime maximal in $\mathfrak{F}$. However, in this case $\operatorname{Ass}(R)$ may consist of more than one ideal. For the example used in Corollary 1.6, the associated primes are $\operatorname{ann}(x)=(2, x, y)$ and $\operatorname{ann}(2)=(2, x)$. This is just one instance where graphs with only two or three vertices prove the exception to the rule.

Proposition 3.2. If $R$ is a ring such that $\left|\Gamma_{E}(R)\right|>3$, then no associated prime of $R$ is a leaf.

Proof. Suppose $[y]$ is an associated prime of degree one. Then there is one and only one $[x]$ such that $[x] \neq[y]$ and $[x][y]=0$. By Lemma $1.2 \operatorname{Ass}(R)=$ $\{\operatorname{ann}(x), \operatorname{ann}(y)\}$. Any additional vertices, of which there are at least two, must be connected to $[x]$. If $[z]$ is another vertex and $\operatorname{deg}[z] \geq 2$, then for some $[w]$, $z w=0 \in \operatorname{ann}(y)$; hence $z \in \operatorname{ann}(y)$ or $w \in \operatorname{ann}(y)$. Since this is not possible, it 
must be that $\operatorname{deg}[z]=\operatorname{deg}[w]=1$. But in order for $[z]$ and $[w]$ to be distinct, we must have $z^{2}=0$ or $w^{2}=0$, which again puts $z \in \operatorname{ann}(y)$ or $w \in \operatorname{ann}(y)$.

Corollary 3.3. If $R$ is a ring such that $\left|\Gamma_{E}(R)\right| \geq 3$, then any vertex with a leaf is an associated prime maximal in $\mathfrak{F}$.

Proof. Proposition 3.1 and Corollary [1.6 take care of the case when there are exactly three vertices. Suppose $\left|\Gamma_{E}(R)\right|>3$. Since every graph is connected, the result follows from Lemma 1.2 and Proposition 3.2 .

We are now in a position to show a finer relationship between vertices of high degree and associated primes.

Proposition 3.4. Let $x_{1}, \ldots, x_{r}$ be elements of $R$, with $r \geq 2$, and suppose $\operatorname{ann}\left(x_{1}\right) \subsetneq \cdots \subsetneq \operatorname{ann}\left(x_{r}\right)$ is a chain in $\operatorname{Ass}(R)$. If $3 \leq\left|\Gamma_{E}(R)\right|<\infty$, then $\operatorname{deg}\left[x_{1}\right]<\cdots<\operatorname{deg}\left[x_{r}\right]$.

Proof. If $\left|\Gamma_{E}(R)\right|=3$, then $|\operatorname{Ass}(R)| \leq 2$ by Lemma 1.2 and Proposition 1.5 and the result follows immediately from Corollaries 1.6 and 3.1. Thus, we may assume that $\left|\Gamma_{E}(R)\right|>3$. By Proposition 2.4. $\Gamma_{E}(R)$ is not a fan graph, given the hypotheses. By the preceding proposition, the degrees of $\left[x_{1}\right]$ and $\left[x_{2}\right]$ are at least two. Suppose $\operatorname{deg}\left[x_{1}\right]=n$. Then there are $n-1$ vertices $\left[u_{i}\right]$, distinct from either $\left[x_{1}\right]$ or $\left[x_{2}\right]$, such that $x_{1} u_{i}=0$ for all $i$. Since $\operatorname{ann}\left(x_{1}\right) \subsetneq \operatorname{ann}\left(x_{2}\right), x_{2} u_{i}=0$ for all $i$ as well. Furthermore, $x_{2} \in \operatorname{ann}\left(x_{1}\right)$, hence $x_{2}^{2}=0$. If $x_{1}^{2} \neq 0$, then each $\operatorname{ann}\left(x_{1}+u_{i}\right)$ contains $x_{2}$ but not $x_{1}$, making $\operatorname{deg}\left[x_{2}\right]>\operatorname{deg}\left[x_{1}\right]$. If $x_{1}^{2}=0$, then take $z \in \operatorname{ann}\left(x_{2}\right) \backslash \operatorname{ann}\left(x_{1}\right)$. Since $x_{1} \notin \operatorname{ann}(z),[z]$ is distinct from $\left[x_{1}\right]$ and $\left[x_{2}\right]$ and connected to $\left[x_{2}\right]$, but not $\left[x_{1}\right]$. Again, $\operatorname{deg}\left[x_{1}\right]<\operatorname{deg}\left[x_{2}\right]$. Moreover, this last argument applies to each pair $\operatorname{ann}\left(x_{i}\right), \operatorname{ann}\left(x_{i+1}\right)$, for $i \geq 1$, since $x_{i} x_{j}=0$ for $1 \leq i \leq j \leq r$.

Example 3.5. Let $R=\mathbb{Z}[X] /\left(X^{3}, X Y\right)$ and let $x$ and $y$ denote the images of $X$ and $Y$, respectively. Then $(x) \subsetneq(x, y)$ is a chain in $\operatorname{Ass}(R)$, corresponding to the vertices $[y]$ and $\left[x^{2}\right]$, respectively, with $\operatorname{deg}[y]=2$ and $\operatorname{deg}\left[x^{2}\right]=3$.

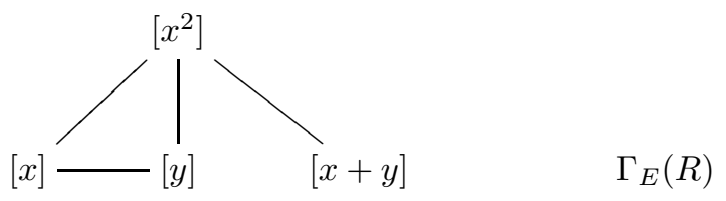

We now make use of Proposition 1.10 to establish a correspondence between associated primes and vertices of relatively large degree.

Theorem 3.6. Let $R$ be such that $2<\left|\Gamma_{E}(R)\right|<\infty$. Then any vertex of maximal degree is maximal in $\mathfrak{F}$ and hence is an associated prime.

Proof. Let $d$ denote the maximal degree of $\Gamma_{E}(R)$. If there is only one vertex of degree $d$, then Proposition 3.1 yields the desired result. Therefore, we may assume that $\left|\Gamma_{E}(R)\right|>3$ and that $\Gamma_{E}(R)$ has at least two vertices of degree $d$. Suppose that $\left[y_{1}\right]$ is a vertex of degree $d$. Then $\operatorname{ann}\left(y_{1}\right) \subseteq \operatorname{ann}\left(y_{2}\right)$ for some $y_{2}$ such that $\operatorname{ann}\left(y_{2}\right)$ is maximal in $\mathfrak{F}$, and so $\left[y_{2}\right]$ is an associated prime. We aim to show that, in fact, $\left[y_{1}\right]=\left[y_{2}\right]$. By Proposition 2.1, $d=\operatorname{deg}\left[y_{1}\right] \leq \operatorname{deg}\left[y_{2}\right] \leq d$, so $\operatorname{deg}\left[y_{2}\right]=\operatorname{deg}\left[y_{1}\right]$. 
Since $\operatorname{ann}\left(y_{1}\right) \subseteq \operatorname{ann}\left(y_{2}\right)$ yields $\left(N\left(\left[y_{1}\right]\right) \backslash\left\{\left[y_{2}\right]\right\}\right) \subseteq\left(N\left(\left[y_{2}\right]\right) \backslash\left\{\left[y_{1}\right]\right\}\right)$, the equality of degrees implies that $\left(N\left(\left[y_{1}\right]\right) \backslash\left\{\left[y_{2}\right]\right\}\right)=\left(N\left(\left[y_{2}\right]\right) \backslash\left\{\left[y_{1}\right]\right\}\right)$; denote this set by $\mathcal{N}$. The connectivity of $\Gamma_{E}(R)$ implies that $d \geq 2$ and so $\mathcal{N} \neq \varnothing$.

To get the desired result, we must show that $\operatorname{ann}\left(y_{1}\right)=\operatorname{ann}\left(y_{2}\right)$, that is, $\operatorname{ann}\left(y_{2}\right) \backslash$ $\operatorname{ann}\left(y_{1}\right)=\varnothing$. Suppose this is not the case and let $z \in \operatorname{ann}\left(y_{2}\right) \backslash \operatorname{ann}\left(y_{1}\right)$. If $[z]$ is distinct from $\left[y_{2}\right]$ and $\left[y_{1}\right]$, then $[z] \in\left(N\left(\left[y_{2}\right]\right) \backslash\left\{\left[y_{1}\right]\right\}\right)$ but $[z] \notin\left(N\left(\left[y_{1}\right]\right) \backslash\left\{\left[y_{2}\right]\right\}\right)$, a contradiction. Suppose $[z]=\left[y_{2}\right]$, in which case $y_{2} y_{1} \neq 0, y_{2}^{2}=0$, and $y_{1}^{2} \neq 0$. Let $[w] \in \mathcal{N}$ and consider $w+y_{2}$, which is annihilated by $y_{2}$, but not $y_{1}$. Then $\left[w+y_{2}\right] \notin \mathcal{N}$ and thus $\left[w+y_{2}\right]=\left[y_{2}\right]$. So if $[v] \in \mathcal{N}$, then $0=\left(w+y_{2}\right) v=w v$. Since $[w]$ was chosen arbitrarily, it follows that the vertices in $\mathcal{N}$ form a complete subgraph. Hence $(\mathcal{N} \backslash\{[w]\}) \cup\left\{\left[y_{2}\right],\left[y_{1}\right]\right\} \subseteq N([w])$ for any $[w] \in \mathcal{N}$, implying that $\operatorname{deg}[w] \geq \operatorname{deg}\left[y_{2}\right]+1$, contradicting the maximality of $\operatorname{deg}\left[y_{2}\right]$.

Suppose $[z]=\left[y_{1}\right]$ and let $[x] \in \mathcal{N}$. If $\operatorname{deg}[x]<d$, then there exists some $u \in$ $\operatorname{ann}\left(y_{1}\right) \backslash \operatorname{ann}(x)$ such that $[u],\left[y_{1}\right]$, and $[x]$ are distinct. Note that $x\left(u+y_{1}\right)=$ $x u \neq 0$, so $\left[u+y_{1}\right] \neq\left[y_{i}\right]$ for $i=1,2$. However, $y_{2}\left(u+y_{1}\right)=0$ which implies $\left[u+y_{1}\right] \in \mathcal{N}$, but $y_{1}\left(u+y_{1}\right)=y_{1}^{2} \neq 0$, contradicting that $\left[u+y_{1}\right] \in \mathcal{N}$. Therefore $\operatorname{deg}[x]=d$. Furthermore, since $y_{2}\left(x+y_{1}\right)=0$ but $y_{1}\left(x+y_{1}\right)=y_{1}^{2} \neq 0$, then $\left[x+y_{1}\right] \in N\left(\left[y_{2}\right]\right) \backslash \mathcal{N}$, hence $\left[x+y_{1}\right]=\left[y_{1}\right]$. Since $[x]$ was chosen arbitrarily in $\mathcal{N}$, it follows that $x_{1} x_{2}=0$ for distinct $\left[x_{1}\right],\left[x_{2}\right] \in \mathcal{N}$. Therefore, the subgraph induced by $\mathcal{N} \cup\left\{\left[y_{2}\right],\left[y_{1}\right]\right\}$ is a complete graph. By Proposition 1.10 there exists a vertex $[w] \in \Gamma_{E}(R)$ such that $\operatorname{deg}[w]<\operatorname{deg}\left[y_{1}\right]$, so $[w] \notin \mathcal{N} \cup\left\{\left[y_{2}\right],\left[y_{1}\right]\right\}$. However, since $\left[y_{2}\right]$ is an associated prime, then by Lemma 1.2, $\operatorname{Ass}(R) \subseteq \mathcal{N} \cup\left\{\left[y_{2}\right],\left[y_{1}\right]\right\}$ and $[w]$ is adjacent to some vertex $[v] \in \mathcal{N} \cup\left\{\left[y_{2}\right],\left[y_{1}\right]\right\}$. This implies that $\operatorname{deg}[v]>d$, a contradiction.

Example 3.7. Recall that $\Gamma_{E}\left(\mathbb{Z}_{4} \times \mathbb{Z}_{4}\right)$ in Example 1.3 had two vertices, namely $[(2,0)]$ and $[(0,2)]$, of maximal degree. Their annihilators are the associated primes of the ring.

Remark 3.8. We collect some comments regarding Theorem 3.6.

(1) The converse is false: For example, in $\mathbb{Z}_{p^{2} q^{3}},\left[p q^{3}\right]$ and $\left[p^{2} q^{2}\right]$ correspond to associated primes $(p)$ and $(q)$, respectively, each maximal in $\mathfrak{F}$, but $\operatorname{deg}\left[p q^{3}\right]=6$ and $\operatorname{deg}\left[p^{2} q^{2}\right]=7$.

(2) The assumption of finiteness is necessary. The graph of the ring $R=$ $\mathbb{Z}[X, Y] /\left(X^{2}, Y^{2}, Z^{2}\right)$ has four vertices with infinite degree, namely $[x y],[x z]$, $[y z]$, and $[x y z]$, but only ann $(x y z)$ is an associated prime of $R$.

Finally, since each of our examples possesses a leaf, one might ask if Corollary 3.3 supersedes Theorem 3.6. that is, if every graph must contain a leaf. Based on the results in section one, the first case where such an example can occur is when the graph has exactly five vertices.

Example 3.9. Let $R=\mathbb{Z}_{3}[X, Y] /\left(X Y, X^{3}, Y^{3}, X^{2}-Y^{2}\right)$, and let $x$ and $y$ denote the images of $X$ and $Y$ in $R$, respectively. 


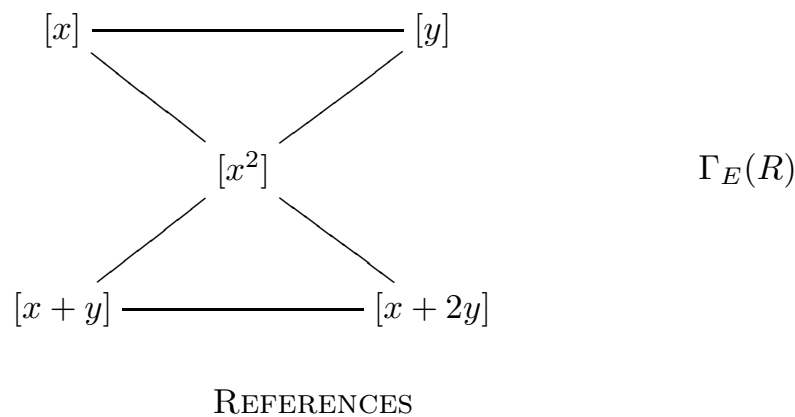

[1] D. Anderson, P. Livingston, The zero-divisor graph of a commutative ring, J. Algebra, 217 (1999) 434-447.

[2] S. Akbari, A. Mohammadian, On the zero-divisor graph of a commutative ring, J. Algebra, 274 (2004) 847-855.

[3] S. Akbari, H.R. Maimani, S. Yassemi, When a zero-divisor graph is planar or a complete r-partite graph, J. Algebra, 270 (2003) 169-180.

[4] I. Beck, Coloring of commutative rings, J. Algebra, 116 (1988), no. 1, 208-226.

[5] H.R. Maimani, M.R. Pournaki, S. Yassemi, Zero-divisor graph with respect to an ideal, Comm. Algebra, 34 (2006) 923-929.

[6] B.R. McDonald, Finite Rings with Identity, Marcel Dekker, Inc., New York, 1974.

[7] S.B. Mulay, Cycles and symmetries of zero-divisors, Comm. Algebra, 30 (2002), no. 7, 35333558 .

[8] S. Redmond, An ideal-based zero-divisor graph of a commutative ring, Comm. Algebra, 31 (2003), no. 9, 4425-4443.

Department of Mathematics, University of Mississippi, Oxford, MS 38677

E-mail address: spiroff@olemiss.edu

Department of Mathematics, Missouri State University, Springfield, MO 65897

E-mail address: cwickham@missouristate.edu 